\title{
Development of a Mental Health Recovery Module for the WHOQOL
}

\section{Melissa J. Rowthorn, D. Rex Billington, Christian U. Krägeloh, Jason Landon, and Oleg N. Medvedev \\ Accepted by Quality of Life Research.}

\begin{abstract}
Purpose The WHOQOL tools are widely used, multi-faceted, patient-rated, quality of life (QoL) measures, developed by the World Health Organisation. The WHOQOL questionnaires are used to assess generic quality of life issues affected by all health problems. This study developed a module to use with the WHOQOL tools to improve their sensitivity to HRQoL issues relevant to mental health recovery.
\end{abstract}

Methods Using a sequential mixed-methods approach, two research stages occurred. A qualitative stage invited 88 participants with experience of mental health recovery into focus groups and importance rating activities to identify candidate items for the new module. Following this, a quantitative stage involved 667 participants with, and without, mental health/addiction issues completing online or paper-based questionnaires to analyse which candidate items differentiated between those with and without mental health/addiction issues. Classical test theory and iterative Partial Credit Rasch Analysis were used to identify the most suitable candidate items for a reliable and valid mental health recovery module to be used with the WHOQOL tools.

Results Seventeen candidate items captured important HRQoL facets relevant to mental health recovery. Rasch analysis removed 10 misfitting items. The final 7-item module, which demonstrated the best Rasch model fit, enquires about: Recovery Beliefs, Identifying Strengths, Self-Awareness, Acceptance, Capacity to Relate, Feeling Understood, and Recovery Progress. Ordinal-to-interval conversion tables have been developed to optimise measurement precision when using the module.

Conclusions Important HRQoL issues central to mental health recovery can be reliably evaluated by using the recovery module with the WHOQOL tools. 


\section{Introduction}

Increasing rates of mental illness and suicide are raising public concern world-wide. The World Health Organization (WHO) reports that $50 \%$ of mental illnesses begin by age 14 , often remain undiagnosed and untreated [1], and over 800,000 people commit suicide each year [2]. The 65th World Health Assembly highlighted that the global burden of mental illness accounts for $25-33 \%$ of all disabilities and requires prioritization for redress [3]. The WHO 2013-2020 mental health action plan asks all countries to review their mental health policies, target factors that lead to poor mental health and commit to reducing rates of suicide and mental illness [2]. To answer this call, there is a need to better understand what leads to the development and progression of mental illness, improved service responsiveness to those at risk of suicide and reliable measures that can identify deterioration in perceived quality of life / progression towards crisis, before people take their own lives.

Quality of life (QoL) can be significantly lower for people with mental illness than for other illnesses [4,5], yet it is highly responsive to intervention, making it a viable treatment target [5-7]. QoL assessment and intervention has been articulated as a way to improve treatment outcomes during mental health service provision for many years [8-17]. Progressive deterioration in subjectively evaluated quality of life has been found to correlate with increased suicide attempts [18,19]. Repeated measurement with QoL tools can identify whether life satisfaction is progressively deteriorating. QoL assessment and intervention are also recognised as ways to improve the effectiveness and relevance of services to people with mental illness [8-10,20]. Yet, without reliable and valid QoL measurement tools, research recommendations are critiqued as hard to implement [21].

Challenges to the development of QoL tools include the evolving nature of the concept and varying ways QoL terminology is used in the literature [22,23]. QoL was defined broadly by the World Health Organisation as: “Individuals' perception of their position in life in the context of the culture and value systems in which they live and in relation to their goals, expectations, standards and concerns. It is a broad ranging concept affected in a complex way by the persons' physical health, psychological state, personal beliefs, social relationships and their relationship to salient features of their environment” (p.291) [8]. This definition is based on the ICF (International Classification of Functioning, Disability and Health) model of health-related QoL [23]. The term health-related quality of life (HRQoL) is recognised as the study of QoL in the context of illness and disease, to help identify how illness/disease affects a person's perception of themselves and their life after the onset of illness and during the course of their recovery [23].

The measurement of recovery is complex; the content of recovery measures also depend upon how recovery is defined. The definitions of mental health recovery vary. They can include clinical 
recovery, personal recovery and stages of recovery definitions [24-26]. Subjective QoL evaluation is closer to the personal recovery definition than to other definitions of recovery [27]. Anthony's [26] definition of recovery is an often-cited definition of personal recovery: “A deeply personal, unique process of changing one's attitudes, values, feelings, goals, skills and roles. It is a way of living a satisfying, hopeful, and contributing life even with limitations caused by the illness. Recovery involves the development of new meaning and purpose in one's life, as one grows beyond the catastrophic effects of mental illness” (p.17).

The 26-item WHOQOL-BREF is a widely-used cross-cultural QoL questionnaire proven to be a valid and reliable patient-rated outcome measure (PROM) for a variety of health treatment programmes [5,8,28-37]. Being generic by design, the WHOQOL tools identify the QoL issues commonly experienced across all health conditions; not QoL issues specific to recovering from a particular health condition [38]. To offset this limitation, condition-specific modules are being developed to use with the WHOQOL tools. These include the HIV [39], Pain [40], OLD [41], AGE [42], Dialysis [43], Disabilities [44], Diabetes [45], and Spirituality, Religiousness, and Personal Beliefs [46] modules. Although, to date, no research into the content for a mental health module has occurred, such a module for the WHOQOL tools would be of value.

Reviews describing: QoL issues specific to mental health recovery [24,47], key determinants of personal recovery [16] and important features of recovery orientated services [27], identify common issues not covered by the content of the WHOQOL tools. The addition of QoL items particularly relevant to recovering from mental illness could improve the WHOQOL's sensitivity and precision as a recovery-orientated PROM for mental health service provision and evaluation purposes. The use of the WHOQOL with a mental health module as a recovery-orientated PROM could support service aims to improve the lives of people recovering from mental health issues. Attention to patterns of deterioration in subjectively rated QoL could also improve the identification of those at risk of suicide within aggregated data if collected by services $[18,19]$.

The aims of this study were to identify the HRQoL issues important to people's recovery from mental illness/addiction; eliminate those identified that are already covered by the WHOQOL-BREF; develop and test the remaining issues as new candidate items; then through psychometric analysis, identify which of these candidate items best measure HRQoL during mental health recovery. The items that meet Rasch measurement criteria will become an adjunct module for the WHOQOL tools. The research was conducted in two interlinked stages. A qualitative focus group stage invited people with mental health recovery experience to identify potential candidate items. This was followed by a quantitative stage to test these candidate items and identify the most suitable items for a reliable and valid mental health recovery module to accompany the WHOQOL tools. The term HRQoL in this 
study refers to the identification of specific QoL issues that emerge in the context of recovering from mental illness/addiction problems.

\section{Method}

\section{Development of Mental Health Recovery Items}

Stage 1 used qualitative methodology informed by PROM and mental health outcome measurement recommendations that stress the importance of using mixed-methods approaches that prioritise end-user and relevant multi-stakeholder involvement when developing mental health outcome and PROM measures [48-50]. The WHO study guidelines for the development of WHOQOL modules [51] and WHOQOL research within New Zealand [52,53] guided the research process and methods.

Table 1 displays the demographic characteristics of the participants contributing to this stage of the research. Two groups of participants participated - those with lived-experience (people identifying as having mental health or addiction problems) and recovery supporters (family or staff). Participants were recruited through Auckland mental health services using purposive and convenience sampling methods. Thirteen focus groups and 11 interviews were conducted with 88 participants to cover a broad scope of service-provision experience and views of minority groups in the mental health and addiction population. Three discussion topics were raised of relevance to the research purpose as vehicles for participants to discuss how QoL changes during mental illness and mental health recovery: 1) what parts of life are most affected by the acute onset of mental illness, 2) what interferes with recovery or assists recovery to progress and 3) what unique QoL issues emerge when recovering from mental illness compared to other health conditions or physical disability? Focus groups and interviews were 60 minutes long and audio recorded.

Following focus group discussions, participants completed two 15-min research activities: 1) rating the importance of existing WHOQOL-BREF facets along with the NZ national items [52] to their personal experience of recovery. The five additional NZ items focused upon the importance of: belonging, meeting the expectations of others, respect, control over one’s life, and managing personal difficulties. 2) Completing a recovery importance exercise: Participants selected personal recovery determinants relevant to their own experiences of recovery from a selection of 48 personal recovery cards. These personal recovery determinants were obtained from a prior review of the personal recovery literature, the content of subjective recovery measures and of key determinants of recovery within seminal reports. Items were prioritised through frequency analysis, then further reduced through thematic analysis and removal of themes already contained within existing items of the NZWHOQOL- 
BREF. Afterwards, light refreshments and vouchers were given to participants in appreciation of their participation.

Table 1 Demographic characteristics of the participants in stage $1(n=88)$.

Demographic category $\quad$ Participant characteristic n

Mental health service users or peer support workers

Family, experts, clinicians / recovery $\quad$ Mental health recovery supporters support workers

Sex

Male $41 \%$

Female $59 \%$

Ethnicity

$\begin{array}{ll}\text { European } & 68 \% \\ \text { Māori } & 12 \% \\ \text { Pasifika } & 2 \% \\ \text { Asian } & 6 \% \\ \text { Other } & 13 \%\end{array}$

Co-existing issues

Physical Health Diagnoses

Mental Health Diagnoses

Mood Disorder

Personality Disorder $4 \%$

Other $2 \%$

\section{$\underline{\text { Psychometric Testing and Module Development }}$}


Stage 2 used quantitative methodology, to test the psychometric properties of the 24 mental health candidate items from Stage 1, to determine the final set of items for an adjunct mental health recovery module. Methodology for item selection was informed by reference to the WHOQOL study protocol for module development [51] and modified criteria for item elimination outlined in the WHOQOLBREF NZ national items study [52]. Supplementary Table S1 specifies the item elimination criterion used to progressively eliminate items to arrive at the best solution for the module.

Adult participants (aged 18+) were recruited New Zealand-wide through convenience and purposive sampling methods. Table 3 displays the demographic characteristics of the 667 Stage 2 participants. Participants were people with lived experience of mental illness/addiction and a comparison group - people identifying as not having had a history of or current experience of these health issues. Participants were equally distributed across four New Zealand regions: Northland, Auckland, Central/Lower North Island, and the South Island. Thirty-five percent of the livedexperience sample were people with mental health diagnoses receiving secondary mental health services, and 65\% self-identified as having an addiction or diagnosed mental illness.

Table 2 Participant characteristics of Stage 2 shown separately for those with lived experiences, the comparison group, and the overall sample $(n=667)$..

\section{Lived}

Demographic Category

\begin{tabular}{lccc}
\hline Number of participants & 389 & 278 & 667 \\
\hline Age Range & & & \\
\hline 18-35 years old & $29 \%$ & $34 \%$ & $31 \%$ \\
$36-50$ years old & $37 \%$ & $27 \%$ & $33 \%$ \\
$51+$ years old & $34 \%$ & $39 \%$ & $36 \%$ \\
Sex (Males) & $33 \%$ & $27 \%$ & $30 \%$ \\
\hline Ethnicity & & & \\
\hline New Zealand European & $72 \%$ & $70 \%$ & $72 \%$ \\
Māori & $21 \%$ & $18 \%$ & $20 \%$ \\
Pasifika & $3 \%$ & $3 \%$ & $3 \%$ \\
Asian & $3 \%$ & $6 \%$ & $4 \%$ \\
Other & $1 \%$ & $2 \%$ & $1 \%$ \\
\hline
\end{tabular}




\begin{tabular}{lccc}
\hline Health issues & & & \\
\hline Physical Health Diagnoses & $30 \%$ & $20 \%$ & $26 \%$ \\
Physical Disabilities & $33 \%$ & $20 \%$ & $28 \%$ \\
\hline Addiction & $30 \%$ & $0 \%$ & $30 \%$ \\
\hline Mental Health Diagnoses & & & \\
\hline Mood Disorder & $66 \%$ & $0 \%$ & $66 \%$ \\
Psychosis & $17 \%$ & $0 \%$ & $17 \%$ \\
Personality Disorder & $7 \%$ & $0 \%$ & $7 \%$ \\
Other & $2 \%$ & $0 \%$ & $2 \%$ \\
\hline
\end{tabular}

Twenty-four candidate items arising out of the qualitative stage of the research were formulated into the Likert-question structure of the existing 31-item NZWHOQOL-BREF survey [53]. Research conducted by Rethfeldt [54] found there was no difference in scoring results when people used an online or paper form of the WHOQOL survey. Two survey formats were created - an online and paper format. To control for order effects, both versions presented $50 \%$ of the surveys with reverse ordered items. The WHOQOL's 'About You' section was modified to ask about current mental health diagnosis, addiction issues, geographic location, co-occurring physical health or disability issues.

\section{$\underline{\text { Data Analysis }}$}

A codebook used in Stage 1 enabled five volunteer listeners to eliminate focus discussion topics referring to existing WHOQOL-BREF or NZWHOQOL national items, identify topics related to the 48 personal recovery determinants used in the importance rating card sort exercise and to record topics not covered by the codebook. The most frequently occurring topics were identified through frequency analysis and forwarded to a question-writing panel. This panel consisted of four QoL, addictions, and mental health researchers. The 26 most frequently discussed topics were further scrutinized by this panel to ensure several criteria were met: items captured the QoL focus required of WHOQOL items, new candidate questions were not covered by existing WHOQOL facets or by other newly developed candidate questions. Referral back to focus group discussions helped to retain the context and meaning of the facet areas, as described by people recovering from mental illness. Questions included some negatively worded items and used question prefixes required by the standardized WHOQOL 
questionnaire format. The final 24 candidate questions with corresponding facet areas are presented in Table 3.

The aims of Stage 1 involved exploring the QoL issues occurring during mental illness to identify potential candidate items. The decision to collect individual data provided an opportunity for all 88 participants to identify QoL factors important to their personal experience of recovery. Triangulation of card sort results with focus group results enabled convergence analysis to establish the most important facets to write questions about.

In Stage 2, descriptive and inferential analyses were completed using SPSS (version 25). Modified New Zealand WHOQOL [52] item-analysis criteria were used to reduce item duplication and to ensure that items differentiated between those with and without mental illness. Remaining items were subjected to Rasch analysis using the software RUMM2030. The purpose of using Rasch analysis was to identify the most suitable items for inclusion in the module based on their measurement capability. Rasch analysis involves testing the order of response option thresholds in polytomous items, item-trait interaction, unidimensionality, local independence assumptions, and potential item bias. Due to the item-trait interaction analysis, Rasch analysis eliminates the need to analyse the role of missing items. Differential item functioning (DIF) analysis identifies where any items perform differently for sub-groups of the sample. When data fit to the Rasch model, these parameters meet the model's expectations, meaning that items can be ordered by difficulty and participants' by ability, on a latent trait (e.g., mental health QoL) using the same log-odds interval scale. This improves a measure's precision to measure what it sets out to measure [55]. Conceptual criteria were considered during iterative Rasch analysis and Classical Test Theory (CTT) steps. Supplementary Table S1 presents the statistical criteria used in this study.

\section{Results}

In stage 1, it was noted that participants in lived experience groups did not speak up as often as participants in recovery supporter groups. Establishing a point of concordance between focus group and card-sort datasets was used to make sure that the relevance of facets highlighted by participants with lived experience in the card-sort exercise was not lost when using frequency analysis across focus groups to identify potential pilot items. Facets discussed in $55 \%$ of focus groups were identified for question writing. Discordant results between focus group and individual exercises revealed differences between participants in the weighting of relevance or importance given to some QoL issues. This strengthened the value of using card-sort data for triangulation and decision-making.

Facets going forward for question writing also included topics discussed in focus groups that were not included in the code book - loneliness, vulnerability, fear of relapse, reciprocity and the needs 
of family members. Table 3 displays the final questions going forward for Stage 2 testing, alongside the focus group themes they represent.

Stage 2 involved testing the performance of these candidate items. Initial analysis showed no evidence of floor or ceiling effects. Kurtosis and skewness were acceptable, with scores between 2.004.00, while the comparison group showed some skewness in candidate items. Twenty-three of the 24 candidate items differentiated between participants with lived experience from those in the comparison group. The candidate item spirituality did not meet this criterion.

The next steps of CTT analysis used the lived-experience sample data, to meet research criterion and construct validity requirements. Conservative conceptual criteria for item elimination were used during CTT steps: an item needed to fail two criterion steps consecutively. If there was a marginal fail, with no strong conceptual reason to discard an item, it was retained to prevent premature elimination of items. MH6-spirituality was eliminated after failing two consecutive criteria. MH22stigma/discrimination and MH23-boredom were identified as correlating less than 0.30 with MH1 (the module's $g$-facet). These items were retained as they correlated with the other construct criterion. Table 4 identifies item correlations against the construct and criterion measures.

Items MH10-health-and-social-services MH13-work readiness, and MH21-social networks were eliminated at CTT step 4. These items correlated highly with existing WHOQOL-BREF items Q18-work, Q22-social support, and Q24-access to healthcare respectively. MH8-coping and MH15hope were eliminated after failing to meet criterion 4 and 5. Both correlated positively with several existing WHOQOL-BREF items: MH8-coping with Q10-energy and Q17-daily living activities; MH15-hope with Q5-enjoying life, Q6-meaningful life, and Q19-satisfaction with oneself. MH8 and MH15 also correlated with existing candidate items: with each other and with MH17-goals. Table 4 displays the remaining 17 QoL items specific to the context of mental-health recovery that do not correlate with existing items in the WHOQOL-BREF or other pilot items.

The 17 mental health QoL candidate items were analyzed through a process of iterative Rasch analysis. This involved the systematic elimination of items producing problematic residual fit or disordered thresholds until an acceptable model fit was evident. Table 5 summarizes the results of the iterative Rasch analysis steps.

The first result: after six misfitting items from the 17 candidate items had been discarded, the chi square statistic became nonsignificant. Yet, there was still evidence of multi-dimensionality. Following the approach used by previous Rasch analysis research, sources of multi-dimensionality were explored through principal components analysis [56]. This revealed a two-factor solution with four items (MH3,7,18,20) heavily cross-loading on a second factor. The three most problematic of these items (MH3,7,20) with cross loadings $>0.4$ were eliminated. 
The second result: An eight-item model, after testing for residual correlations revealed local dependency between items MH14 and MH4. MH14 had been intermittently red flagged for disordered threshold throughout the analysis, so it was chosen for removal. This resolved the local dependency problem. The third result, a seven-item version, showed the best model fit, strict unidimensionality, with no evidence of differential item functioning (DIF) by demographic factors. Table 6 displays the content of the final seven-item module, together with the initial item-related Stage 1 focus group themes they correspond to.

Figure 1 displays the person-item threshold distribution including extreme persons for the final module. This figure indicated good targeting of the sample by item thresholds with an adequate spread of item difficulty, allowing for the assessment of individuals' QoL across the whole continuum of possible responses for each item

Fig. 1 Person-item threshold distribution (including extreme scores) for the final module displaying mean and standard deviation

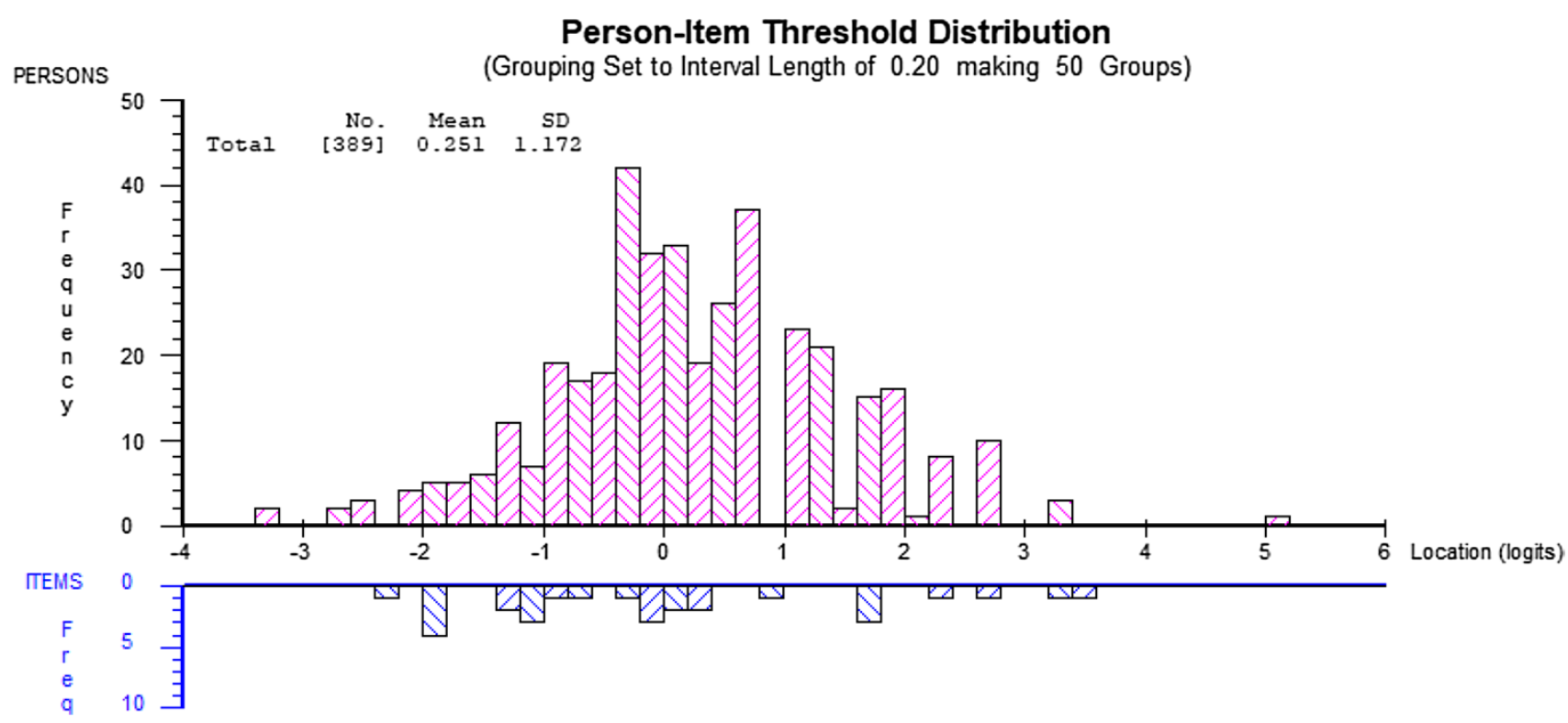




\section{Insert Supplementary Table S1}

Supplementary Table S1 - Methodological steps and criteria used for item elimination.

\section{Steps Methodological aim}

\section{Criterion description}

Step 1 Skewedness of each item is assessed

Step 2 Discriminant validity of each item is assessed

Step 3 Construct validity of each item is assessed

Step 4 Ensure no duplication with existing WHOQOL-BREF items

Step 5 Ensure no duplication with existing candidate items

Step 6 Rasch criteria for item inclusion assessed
The item's mean is between 2.00-4.00 on the 5-point Likert scale. There are no obvious ceiling or floor effects.

Kurtosis scores are between -1.00 and +1.00 .

Items discriminate between those with and without mental health and addiction issues. Any item with a result of $p>0.05$ (Mann Whitney $\mathrm{U}$ test) is red-flagged for elimination, if failing the next criteria.

Using the mental health data set only - item's correlation (Spearman's rho) above 0.30 with 1) Total QOL 2) the $g$ facet 3) MH $g$ Facet, and 4) MH1.

Spearman's item correlations are below 0.60 with any existing WHOQOL item.

Spearman’s item correlations are below 0.60 with any existing mental health candidate items.

Items are removed if identified as having disordered thresholds, differential item functioning in any demographic characteristics (DIF), or evidence of local dependency - if item residual correlations were $>0.2$ above the mean of all residual correlations. The final model needs to demonstrate nonsignificant chi square and a Person Separation Index (PSI) above 0.80 when extreme persons are included, to be used for individual and group analysis. Rasch unidimensionality analysis needs to show acceptable fit to Rasch expectations. Person-Item threshold distribution, with extreme persons included, needs to show an acceptable item difficulty distribution and measurement capability across the sample.

Conceptual criteria are met throughout

Practicality of items for redress within mental health service environments, question complexity considered, items are aligned with the WHO definitions of HRQoL and mental health. Items can be used in aggregated or individual recovery planning applications.

Module length is kept as short as possible without losing reliability/validity for subsamples of the population. 
Items are sensitive to change and relevant to the subjective processes involved with recovering one's life following mental health/addiction problems. 
Table 3 HRQoL candidate items and related mental health recovery facets identified in the Stage 1 focus groups.

Pilot questions to be tested in Stage 2

MH 1 How would you rate your mental health?

MH 2 To what extent do you feel lonely?

MH 3 To what extent have you made changes for the better?

MH 4 To what extent do you believe you can recover from illness?

MH 5 To what extent do you recognise positive strengths you have?

MH 6 To what extent do spiritual values help guide your life?

MH 7 To what extent are you determined to recover?

MH 8 To what extent do you feel you are able to cope with daily life?

MH 9 To what extent does understanding why you have a problem help you to resolve it?

MH 10 To what extent do the health and social services meet your needs?

MH 11 How well have you been able to adjust to what has happened in your life?

MH 12 How well do you feel you can relate to others?

MH 13 To what extent do you consider that you are fit and able to work?

MH 14 How confident are you that when well, you can stay well?

MH 15 To what extent do you feel positive about your future?

MH 16 To what extent are you motivated by people who have overcome similar problems that you have?

MH 17 To what extent are you confident you can attain your goals in life?

MH 18 How satisfied are you that people really understand you?

MH 19 How satisfied are you with your recovery?

MH 20 How satisfied are you that other people recognise your strengths?

MH 21 How satisfied are you that there are people there for you when you need them?

MH 22 How much do you feel discriminated against?

MH 23 How frequently do you feel bored?

MH 24 How satisfied are you with the amount of physical activity you engage in?

\section{Focus group facets}

Mental Health/Illness symptoms

Loneliness

Personal Growth/Transformation/Healing

Belief in Recovery

Recognising Strengths

Spirituality

Determination/Recovery Readiness

Coping Skills/Resilience/Vulnerability

Self-Awareness/Insight

Unmet needs/Alternative Healthcare

Acceptance/Adjustment

People Skills/Relating to others/Connection

Work Readiness

Fear of Relapse/Confidence

Hope

Peer Support

Goal achievement/Uncertain Future

Understanding/Empathy

Recovery Progress

Recognising Strengths

Social Networks

Stigma/Discrimination

Having things to do

Exercise 
Table 4 - Results of the 17 items remaining after CTT analysis - item correlations* with HRQoL, relevant G items, MH status

\begin{tabular}{|c|c|c|c|c|c|c|}
\hline Item & Question & $\begin{array}{l}\text { Mean Total } \\
\text { HRQoL score }\end{array}$ & G1+G2 & G1+MH1 & G1 & MH1 \\
\hline MH 2 & To what extent do you feel lonely? & $.55^{* *}$ & $.43^{* *}$ & $.49^{* *}$ & $.49^{* *}$ & $.39^{* *}$ \\
\hline MH 3 & To what extent have you made changes for the better? & $.45^{* *}$ & $.39^{* *}$ & $.41^{* *}$ & $.38^{* *}$ & $.32^{* *}$ \\
\hline MH 4 & To what extent do you believe you can recover from illness? & $.58^{* *}$ & $.46^{* *}$ & $.54^{* *}$ & $.45^{* *}$ & $.49^{* *}$ \\
\hline MH 5 & To what extent do you recognise the positive strengths you have? & $.59^{* *}$ & $.46^{* *}$ & $.54^{* *}$ & $.46^{* *}$ & $.48^{* *}$ \\
\hline MH 7 & To what extent are you determined to recover your health when not well? & $.46^{* *}$ & $.35^{* *}$ & $.40^{* *}$ & $.34^{* *}$ & $.35^{* *}$ \\
\hline MH 9 & To what extent does understanding why you have a problem help you to resolve it? & $.47^{* *}$ & $.32^{* *}$ & $.39^{* *}$ & $.34^{* *}$ & $.34^{* *}$ \\
\hline MH 11 & How well have you been able to adjust to what has happened in your life? & $.61^{* *}$ & $.48^{* *}$ & $.52^{* *}$ & $.49^{* *}$ & $.44^{* *}$ \\
\hline MH 12 & How well do you feel you can relate to others? & $.45^{* *}$ & $.32^{* *}$ & $.45^{* *}$ & $.36^{* *}$ & $.42^{* *}$ \\
\hline MH 14 & How confident are you that when well, you can stay well? & $.60^{* *}$ & $.49^{* *}$ & $.60^{* *}$ & $.50^{* *}$ & $.55^{* *}$ \\
\hline MH 16 & $\begin{array}{l}\text { To what extent are you motivated by people who have overcome similar problems that } \\
\text { you have? }\end{array}$ & $.39^{* *}$ & $.32^{* *}$ & $.37^{* *}$ & $.35^{* *}$ & $.31^{* *}$ \\
\hline MH 17 & To what extent are you confident you can attain your goals in life? & $.65^{* *}$ & $.54^{* *}$ & $.60^{* *}$ & $.57^{* *}$ & $.48^{* *}$ \\
\hline MH 18 & How satisfied are you that people really understand you? & $.65^{* *}$ & $.52^{* *}$ & $.58^{* *}$ & $.48^{* *}$ & $.54^{* *}$ \\
\hline MH 19 & How satisfied are you with your recovery? & $.64^{* *}$ & $.53^{* *}$ & $.62^{* *}$ & $.49^{* *}$ & $.59^{* *}$ \\
\hline MH 20 & How satisfied are you that other people recognise your strengths? & $.54^{* *}$ & $.38^{* *}$ & $.43^{* *}$ & $.39^{* *}$ & $.36^{* *}$ \\
\hline MH 22 & How often do you feel discriminated against? & $.38^{* *}$ & $.32^{* *}$ & $.32^{* *}$ & $.32^{* *}$ & $.23^{* *}$ \\
\hline MH 23 & How frequently do you feel bored? & $.38^{* *}$ & $.35^{* *}$ & $.35^{* *}$ & $.36^{* *}$ & $.26^{* *}$ \\
\hline MH 24 & How satisfied are you with the amount of physical activity you engage in? & $.45^{* *}$ & $.48^{* *}$ & $.39^{* *}$ & $.32^{* *}$ & $.35^{* *}$ \\
\hline
\end{tabular}

Note: Bolded items are the items referred to in Table 5, as the first result.

* Spearman's rho >.30 with 1) Total QOL 2) the $g$ facet (G1+G2), 3) MH $g$ Facet (G1+MH1) \& 4) G1 \& 5) MH1 where G items are the general items from the WHOQOL-BREF and the general item from the candidate item selection MH1 - how satisfied are you with your mental health? ** $p<.01$ 
Table 5 The overall Rasch model fit statistics for the iterative module development steps.

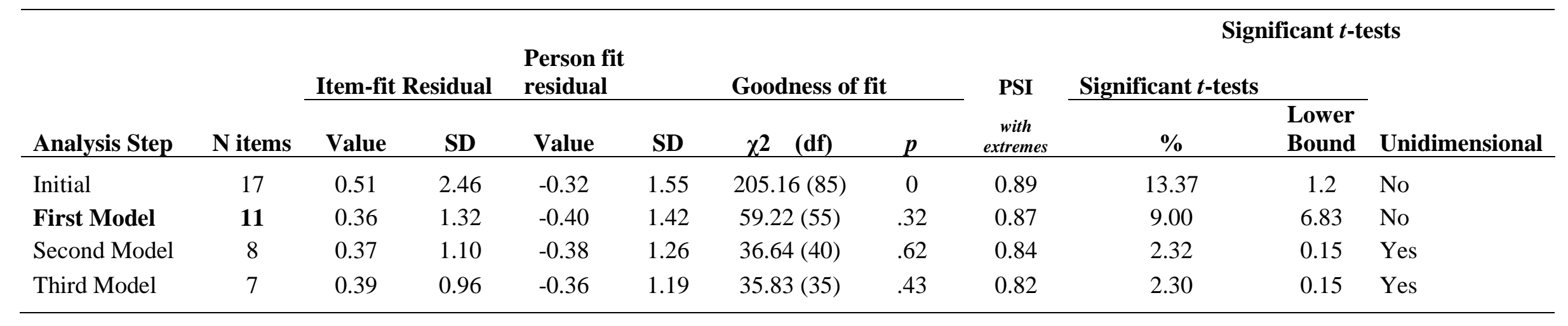

Table 6 Final Mental Health Recovery Module Questions

\begin{tabular}{lll}
$\begin{array}{l}\text { Module } \\
\text { Item }\end{array}$ & Module Question & Focus Group Theme \\
\hline MH 4 & To what extent do you believe you can recover from illness? & Belief in Recovery \\
MH 5 & To what extent to do you recognise the positive strengths you have? & Identifying Strengths \\
MH 9 & To what extent does understanding why you have a problem help you to resolve it? & Insight/Self-Awareness \\
MH 11 & How well have you been able to adjust to what has happened? & Acceptance/Adjustment \\
MH 12 & How well do you feel you can relate to others? & People Skills/Connection \\
MH 18 & How satisfied are you that people really understand you? & Understanding/Empathy \\
MH 19 & How satisfied are you with your recovery? & Recovery progress \\
\hline
\end{tabular}


Meeting expectations of the unidimensional Rasch model permits generation of ordinal-to-interval conversion tables to transform ordinal raw scores into interval-level data. Table 7 provides conversion scores for the module using both logits and scale metrics for convenience. Module users can refer to this conversion table, to increase measurement precision, by simply finding the corresponding interval-level score for the person's raw (ordinal scale) score on the right-hand side of the table.

Table 7 Ordinal-to-interval conversion scores for the 7-item mental health recovery module.

\begin{tabular}{|c|c|c|}
\hline \multirow{2}{*}{$\begin{array}{c}\text { Ordinal } \\
\text { Scale }\end{array}$} & \multicolumn{2}{|c|}{ Interval } \\
\hline & Logit & Scale \\
\hline 7 & -4.18 & 7.00 \\
\hline 8 & -3.36 & 9.49 \\
\hline 9 & -2.80 & 11.21 \\
\hline 10 & -2.41 & 12.39 \\
\hline 11 & -2.11 & 13.31 \\
\hline 12 & -1.85 & 14.09 \\
\hline 13 & -1.63 & 14.77 \\
\hline 14 & -1.43 & 15.38 \\
\hline 15 & -1.24 & 15.95 \\
\hline 16 & -1.06 & 16.49 \\
\hline 17 & -0.89 & 17.02 \\
\hline 18 & -0.72 & 17.53 \\
\hline 19 & -0.56 & 18.04 \\
\hline 20 & -0.39 & 18.55 \\
\hline 21 & -0.22 & 19.07 \\
\hline 22 & -0.04 & 19.61 \\
\hline 23 & 0.15 & 20.18 \\
\hline 24 & 0.34 & 20.77 \\
\hline 25 & 0.55 & 21.41 \\
\hline 26 & 0.78 & 22.10 \\
\hline 27 & 1.02 & 22.86 \\
\hline 28 & 1.30 & 23.69 \\
\hline 29 & 1.61 & 24.63 \\
\hline 30 & 1.95 & 25.69 \\
\hline 31 & 2.34 & 26.88 \\
\hline 32 & 2.80 & 28.26 \\
\hline 33 & 3.34 & 29.92 \\
\hline 34 & 4.06 & 32.11 \\
\hline 35 & 5.01 & 35.00 \\
\hline
\end{tabular}

\section{Discussion}


This research aimed to develop a mental health recovery module for the WHOQOL tools to better assess the HRQoL of people recovering from mental health and addiction issues. The developed module demonstrates excellent reliability, internal consistency and unidimensionality for measuring HRQoL issues specific to mental health recovery. The module met expectations of the Rasch measurement model permitting production of conversion tables that can be used to improve assessment accuracy by transforming individual raw scores into interval-level data suitable for parametric tests. The module items showed invariance across all demographic groups with no significant DIF.

Seven of the initial 24 HRQoL items identified from Stage 1 (including items about hope, coping, and spirituality) did not meet CTT inclusion criteria because they were covered by the existing WHOQOL-BREF or final module items. A review of qualitative studies into mental health recovery described hopelessness, for example, as not having a positive future, poor coping, lost aspirations and not moving forward [16]. The current study found hope correlated with the existing WHOQOL item identifying hope in the facet descriptor (Q5-enjoyment of life) as well as with the candidate items MH17-goals and MH8-coping. The module item MH19 (recovery progress) is a tangible measure of perceived progress, or moving forward, which can have an impact upon hope in ways that can be targeted within mental health and addiction services.

The 17 items meeting CTT criteria are all relevant HRQoL issues to personal recovery. These 17 items could be included in item-banks as discussion items for this population. The seven items remaining after Rasch analysis were more sensitive measures of QoL in the context of mental health recovery that worked well across all participants and demonstrated the best measurement capability. The module's content aligns with findings from reviews of the personal recovery literature that identify self and relational factors being of importance to recovery that are inclusive of acceptance, understanding from others, belief in recovery, a focus on strengths, personal adjustment and a recovery orientation meaningful to the person concerned $[16,27,57,58]$.

When PROMs are used as discussion and recovery planning tools, service providers can explore the relevance of facet areas to service users, address issues of importance within individualized recovery plans and identify issues impeding or supporting a person's recovery [59]. Each person recovering from mental illness or addiction may present with a different cluster of affected HRQoL facets identified within the module, the WHOQOL, or through a combination of both measures. Aggregated use of PROM data empowers the service-user voice within outcome measurement suites and offers service users an opportunity to set the recovery direction of service delivery in collaboration with service providers. This is outlined by service users as important within 
service delivery and outcome measurement processes [60].

The final module includes perceived subjective and perceived objective items central to the WHOQOL's measurement of QoL [38] and items highlighted as important determinants of mental health recovery by SAMHSA in the areas of hope, strengths, self-direction, non-linear pathways, relational, and respect components [58]. Potential ways to use the WHOQOL and adjunct module as a PROM to improve service delivery could involve discussion about engagement and the therapeutic alliance when assessing changes within the relational questions of the WHOQOL and adjunct module. A service's recovery orientation could be evaluated by discussing and examining facets that cover issues relevant to recovery and to the seven mental health outcome domains recommended for inclusion in outcome measurement suites [49].

\section{Limitations}

Mood disorders, as one demographic category, prevented the weighting of depression versus other mood disorders on study findings. The research sample was not a representative sample of the New Zealand primary, secondary or tertiary mental health or addiction consumer population. Purposeful sampling did involve recruiting from primary and secondary mental health and addiction settings across New Zealand with opportunities for convenience and purposeful sampling aimed at improving Māori representation. Participants were in mid to late phases of mental health recovery, with more participants in the $>45$ age group. There was no targeted recruitment of participants from acute inpatient, deaf mental health or forensic services. Module questions are worded for use as a generic recovery tool for a population with a diverse health profile. Use of the tool would require instruction to focus the question about recovery progress upon the specific health issue the service is treating at the time of using the measure. The module is a standalone measure for scoring purposes. It is designed, however, to be used with the WHOQOL to ensure that both generic and specific HRQoL issues relevant and important to mental health recovery are captured during HRQoL assessment and reviews.

The module has yet to be assessed for test-retest reliability. The five New Zealand national items [52] were included to allow for the socio-cultural context of QoL to New Zealand participants. These items may be relevant to people recovering from mental illness in other countries as they are focused upon psychological and social domains. Although no DIF was noted by cultural demographics, Pasifika and Asian cultures were under-represented in the sample based on New Zealand population statistics. The module has yet to be validated in other countries.

\section{Conclusions}


This research aimed to develop a module to measure HRQoL issues important to the process of recovering from mental illness. The module was developed with end users in alignment with WHOQOL module development and mental health recovery principles and has been satisfactorily validated using both CTT and Rasch analysis.

The module can be used with the WHOQOL measures to improve their sensitivity as PROMs to track changes in the HRQoL of people recovering from mental illness. The use of this module may contribute to improved understanding and more comprehensive assessment of HRQoL facets specific to recovering from mental illness/addiction problems. Studies have shown that progressively deteriorating scores within HRQoL assessments can identify service users with unmet rehabilitation needs, requiring treatment reviews, or at increased risk of suicide $[6,9,10,18,19]$. The WHOQOL with the adjunct module can be used to identify facets important to service users to address during treatment and can be used as a service level outcome measure to explore deterioration or improvement in HRQoL during treatment or service provision. Services interested in improving the recovery orientation of their service can use the module with the WHOQOL-BREF as a PROM for quality improvement and service evaluation purposes. Future research may identify whether some items within the WHOQOL tools are more significant than others to people recovering from mental illness/addiction. If so, those items, along with these module items, could be used to develop a briefer HRQoL measure for this population.

\section{Compliance with Ethical Standards:}

Funding: No grants or external funding were involved in this research. All authors declare that they have no conflicts of interest.

Ethical approval: This article does not contain any studies with animals performed by any of the authors. All procedures performed in studies involving human participants were reviewed and approved by the Auckland University of Technology Ethics Committee (AUTEC approval reference 14/227) which is aligned with New Zealand National Ethics Committee guidelines and the 1964 ethical standards within the Helsinki declaration and its later amendments.

Agencies were approached and gave consent to invite the people receiving and working in their services into the study. Participants with lived experience of mental illness were in stable states of mental health, identifying themselves as being in mid-later stages of recovery. All participants were provided with information sheets detailing the purposes of the study, activities involved, their rights 
to withdraw, contact details to request further information about the study, consent forms, and opportunities to ask questions with staff and of the researcher. Support was available to participants during and following research activities. Participants had a choice of participating in group or individual interviews in Stage 1, or of completing online or paper versions of the survey in Stage 2, as well as choices to enter a grocery voucher draw or to receive information about the study at its conclusion, if desired. All participants self-identified themselves as capable of participating in the study by signing paper-based consent forms in both studies or by clicking on a progress to the survey link, after reading the online information sheet, if they consented to participate in the online version of the questionnaire in Stage 2. Participants knew they could withdraw at any time with no consequences to them in both studies. 


\section{References}

1. World Health Organisation. (2018, October,10). Retrieved from URL: https://www.who.int/mental_health/world-mental-health-day/2018/en/

2. World Health Organisation (WHO), Global Status Report Mental Health Action Plan 20132020, NLM classification: WM 101 ISBN: 978-92-4-150602-1. Retrieved from URL: http://apps.who.int/iris/bitstream/handle/10665/89966/9789241506021_eng.pdf?sequence=1

3. World Health Organisation. (2012). Agenda item 13.2 WHA65.4: The global burden of mental disorders and the need for a comprehensive, coordinated response from health and social sectors at country level. The 65th World Health Assembly, 9th Plenary Meeting, 25th May, A65/VR/9. Retrieved from URL: https://www.who.int/mental_health/WHA65.4_resolution.pdf

4. Akvardar, Y., Akdede, B.B., Ozerdem, A., Eser, E., Topyaka, S., \& Alptekin, K. (2006) Assessment of quality of life with the WHOQOL-BREF in a group of Turkish psychiatric patients compared with diabetic and healthy subjects. Psychiatry Clinical Neuroscience, 60(6), 693-699. https://doi.org/10.1111/j.1440-1819.2006.01584.x

5. Skevington, S.M., \& McCrate, F.M. (2011). Expecting a good quality of life in health: assessing people with diverse diseases and conditions using the WHOQOL-BREF. Health Expectations, 15(1), 49-62. https://doi.org/10.1111/j.1369-7625.2010.00650.x

6. Revicki, D.A., Kleinman, L. \& Cella, D. (2014). A history of health-related quality of life outcomes in psychiatry. Dialogues in Clinical Neuroscience, 16(2), 127-135. PMID: 25152652

7. Köhler, S., Unger, T., Hoffman, S., Mackert, A., Ross, B., Fydrich, T. (2015). The relationship of health-related quality of life and treatment outcome during inpatient treatment of depression. Quality of Life Research, 24(3), 641-9. https://doi.org/10.1007/s11136-014-0811-8

8. Orley, J., Saxena, S., \& Herrman, H. (1998). Quality of life and mental illness: Reflections 
from the perspective of the WHOQOL. The British Journal of Psychiatry, 172(4), 291-293. https://doi.org/10.1192/bjp.172.4.291

9. Royal College of Psychiatrists. (1999). Predictors of quality of life in people with severe mental illness. Study methodology with baseline analysis in the UK700 trial. The British Journal of Psychiatry, 175(5), 426-432. https://doi.org/10.1192/bjp.175.5.426

10. Prince, P. N. \& Prince, C. R. (2001). Subjective quality of life in the evaluation of programs for people with serious and persistent mental illness. Clinical Psychology Review, 21(7), 1005-36. https://doi.org/10.1016/S0272-7358(00)00079-9

11. Larsen, T.K, Melle, I., Friis, S., Haahr, U., Johannesen, J. O., Larsen, T. K., Opjordsmoen, S., \& McGlashan, T. (2005). Measuring quality of life in first-episode psychosis. European Psychiatry, 20(7), 474-483. https://doi.org/10.1016/j.eurpsy.2005.03.002

12. Masthoff, E. D., Trompenaars, F. J., Van Heck, G. L., Hodiamont, P. P., \& De Vries, J. (2006b). Quality of life and psychopathology: investigations into their relationship. Australian and New Zealand Journal of Psychiatry, 40(4): 333-340. https://doi.org/10.1080/j.1440-1614.2006.01799.x

13. Masthoff, E. D., Trompenaars, F. J., Van Heck, G. L., De Vries, J., \& Hodiamont, P. P. (2006a). The relationship between stress and quality of life in psychiatric outpatients. Stress and Health, 22(4), 249-255. https://doi.org/10.1002/smi.1105

14. Bayliss, M., Rendas-Baum, R., White, M.K., Maruish, M., Bjorner, J. \& Tunis, S.L. (2012). Health-related quality of life (HRQL) for individuals with self-reported chronic physical and/or mental health conditions: panel survey of an adult sample in the United States. Health and Quality of Life Outcomes, 10:154-64. https://doi.org/10.1186/1477-7525-10-154

15. Renwick, L., Jackson, D., Foley, S., Owens, E., Ramperti, N., Behan, C., O'Callaghan, E. (2012). Depression and quality of life in first-episode psychosis. Comprehensive Psychiatry, 53(5), 451-455. https://doi.org/10.1016/j.comppsych.2011.07.003

16. Connell, J., Brazier, J., O'Cathain, A., Lloyd-Jones, M., \& Paisley, S. (2012). Quality of life 
of people with mental health problems: a synthesis of qualitative research. Health and Quality of Life Outcomes, 10:138-169. https://doi.org/10.1186/1477-7525-10-138

17. Medici, C. R., Vestergaard, C. H., Hjorth, P., Hansen, M. V., Shanmuganathan, J. W., Viuff, A. G., \& Munk-Jørgensen, P. (2016). Quality of life and clinical characteristics in a nonselected sample of patients with schizophrenia. International Journal of Social Psychiatry, 62(1), 12-20. https://doi.org/10.1177/0020764015585330

18. Ponizovsky, A.M., Grinshpoon, A., Levav, I., \& Ritsner, M.S. (2003). Life satisfaction and suicidal attempts among persons with schizophrenia. Comprehensive Psychiatry, 44(6), 442447. https://doi.org/10.1016/S0010-440X(03)00146-9

19. De Abreu, L.N., Nery, F.G., Harkavy-Friedman, J.M., de Almeida, K.M., Gomes, B.C., Oquendo, M.A., \& Lafer, B. (2012). Suicide attempts are associated with worse quality of life in patients with bipolar disorder type I. Comprehensive Psychiatry, 53(2), 125-129. https://doi.org/10.1016/j.comppsych.2011.03.003

20. Awad, G.A. (2016). The Concept of Quality of Life in Schizophrenia: From “An Ethereal Entity”' to a Valued Health Outcome. Journal of Psychosocial. Rehabilitation Mental Health, 3(2), 51-52 https://doi.org/10.1007/s40737-016-0062-9

21. Katschnig H. (2006). Quality of life in mental disorders: challenges for research and clinical practice. World Psychiatry, 5(3), 139-45. Retrieved from URL: https://www.ncbi.nlm.nih.gov/pmc/articles/PMC1636133/

22. Karimi, M. and Brazier, J. (2016). Health, Health-Related Quality of Life, and Quality of Life: What's the Difference? Pharmacoeconomics, 34(7), 645-9. https://doi.org/10.1007/s40273-016-0389-9

23. Bakas, T., McLennon, S.M., Carpenter, J.S. Buelow, J.M., Otte, J.L., Hanna, K.M. “...” Welch, J.L. (2012). Systematic review of health-related quality of life models. Health and Quality of Life Outcomes, 10(1), 134-46. https://doi.org/10.1186/1477-7525-10-134

24. Keetharuth A.D., Brazier, J., Connell, J., Bjorner, J.B., Carlton, J., Taylor-Buck, E., Ricketts, T., McKendrick, K., Browne, J., Croudace, T., Barkham, M. (2018). Recovering 
Quality of Life (ReQoL): a new generic self-reported outcome measure for use with people experiencing mental health difficulties. British Journal of Psychiatry, 212(1), 42-49. https://doi.org/10.1192/bjp.2017.10

25. Onken, S.J., Craig, C.M., Ridgway, P., Ralph, R.O., \& Cook, J.A. (2007). An analysis of the definitions and elements of recovery: A review of the literature. Psychiatric Rehabilitation Journal, 31(1), 9-22. https://doi.org/10.2975/31.1.2007.9.22

26. Anthony, W. (1993). Recovery from mental illness: the guiding vision of the mental health system in the 1990’s. Psychosocial Rehabilitation Journal, 16(4), 11-23. http://dx.doi.org/10.1037/h0095655

27. Slade, M. (2013). 100 ways to support recovery: a guide for mental health professionals, $2^{\text {nd }}$ ed., Rethink, London. Retrieved from URL: https://www.rethink.org/adviceinformation/living-with-mental-illness/treatment-and-support/100-ways-to-supportrecovery/

28. Masthoff, E.D., Trompenaars, F.J., Van Heck, G.L., Hodiamont, P.P., \& De Vries, J. (2005). Validation of the WHO Quality of Life assessment instrument (WHOQOL-100) in a population of Dutch adult psychiatric outpatients. European Psychiatry, 20(7), 465-473. https://doi.org/10.1016/j.eurpsy.2004.09.012

29. Trompenaars, F.J., Masthoff, E.D., Van Heck, G.L., Hodiamont, P.P., \& De Vries, J. (2006). The WHO Quality of Life Assessment Instrument (WHOQOL-100) - Investigating its discriminant ability for psychiatric outpatients. European Journal of Psychological Assessment, 22(3), 207-215. https://doi.org/10.1027/1015-5759.22.3.207

30. Trompenaars, F.J., Masthoff, E.D., Van Heck, G.L., Hodiamont, P.P., \& De Vries, J. (2006c). Relationship between mood-related disorders and quality of life in a population of Dutch adult psychiatric outpatients. Depression and Anxiety, 23(6), 353-363. https://doi.org/10.1002/da.20180

31. Mas-Exposito, L., Amador-Campos, J.A., Gomez-Benito, J., \& Lalucat-Jo, L. (2011). The World Health Organization Quality of Life Scale Brief Version: a validation study in 
patients with schizophrenia. Quality of Life Research, 20(7), 1079-1089.

https://doi.org/10.1007/s11136-011-9847-1

32. Berlim, M., Pavanello, D., Caldieraro, M., \& Fleck, M.P. (2005). Reliability and validity of the WHOQOL-BREF in a sample of Brazilian outpatients with major depression. Quality of Life Research, 14(2), 561-564. https://doi.org/10.1007/s11136-004-4694-y

33. Garcia-Rea, E.A., \& LePage, J.P. (2010). Reliability and validity of the World Health Organization quality of life: brief version (WHOQOL-BREF) in a homeless substance dependent veteran population. Social Indicators Research, 99(2), 333-340. https://doi.org/10.1007/s11205-010-9583-x

34. De Vries, J., \& Van Heck, G.L. (1994). Quality of Life and Refugees. International Journal of Mental Health, 23(3), 57-75. https://doi.org/10.1080/00207411.1994.11449287

35. Oliveira, S. E.H., Carvalho, H., \& Francisco Esteves, F. (2016). Toward an understanding of the quality of life construct: Validity and reliability of the WHOQOL-BREF in a psychiatric sample. Psychiatry Research, 244:37-44. https://doi.org/10.1016/j.psychres.2016.07.007

36. Priebe, S., Reininghaus, U., McCabe, R., Burns, T., Eklund, M., Hansson, L., Wang, D. (2010). Factors influencing subjective quality of life in patients with schizophrenia and other mental disorders: a pooled analysis. Schizophrenia Research, 121(1-3), 251-258. https://doi.org/10.1016/j.schres.2009.12.020

37. Rocha, N.S., Power, M.J., Bushnell, D.M., \& Fleck, M.P. (2012). Cross-cultural evaluation of the WHOQOL-BREF domains in primary care depressed patients using Rasch analysis. Medical Decision Making, 32(41), 41-55. https://doi.org/10.1177/0272989X11415112

38. Skevington, S.M., Sartorius, N., \& Amir, M. (2004). Developing methods for assessing quality of life in different cultural settings. The history of the WHOQOL instruments. Social Psychiatry and Psychiatric Epidemiology, 39(1), 1-8. https://doi.org/10.1007/s00127-004$\underline{0700-5}$

39. (2003) Initial steps to developing the World Health Organization's Quality of Life 
Instrument (WHOQOL) module for international assessment in HIV/AIDS. AIDS Care, 15(3), 347-357. https://doi.org/10.1080/0954012031000105405

40. Mason, V.L., Skevington, S.M., \& Osborn, M. (2004). Development of a Pain and Discomfort Module for use with the WHOQOL 100. Quality of Life Research, 13(6), 11391152. https://doi.org/10.1023/B:QURE.0000031344.53009.eb

41. Power, M., Quinn, K. \& Schmidt, S., \& the WHOQOL OLD Group. (2005). Development of the WHOQOL-Old Module. Quality of Life Research, 14(10), 2197-2214. https://doi.org/10.1007/s11136-005-7380-9

42. Caballero, F.F., Miret, M., Power, M., Chatterji, S., Tobiasz-Adamczyk, B., Koskinen, S., Leonardi, M., Olaya, B., Haro, J.M., \& Ayuso-Mateos, J.L. (2013). Validation of an instrument to evaluate quality of life in the aging population: WHOQOL-AGE. Health and Quality of Life Outcomes, 11:177. https://doi.org/10.1186/1477-7525-11-177

43. Yang, S.C., Kuo, P.W., Wang, J.D., Lin, M.I., \& Su, S. (2006). Development and psychometric properties of the dialysis module of the WHOQOL BREF Taiwan version. Journal of Formosan Medical Association, 105(4), 299-309. https://doi.org/10.1016/S0929$\underline{6646(09) 60121-2}$

44. Power, M.J., Green, A.M. \& The WHOQOL-DIS GROUP (2010). Development of the WHOQOL disability module. Quality of Life Research, 9(4), 571-584. https://doi.org/10.1007/s11136-010-9616-6

45. Lin, C. Y., Lee, T. Y., Sun, Z. J., Yang, Y. C., Wu, J. S., \& Ou, H. T. (2017). Development of diabetes-specific quality of life module to be in conjunction with the World Health Organization quality of life scale brief version (WHOQOL-BREF). Health and Quality of Life Outcomes, 15(1), 167. https://doi.org/10.1186/s12955-017-0744-3

46. Skevington, S.M., Gunson, K.S., O’Connell, K.A. (2013). Introducing the WHOQOL-SRPB BREF: developing a short-form instrument for assessing spiritual, religious and personal beliefs within quality of life. Quality of Life Research, 22(5), 1073-1083. https://doi.org/10.1007/s11136-012-0237-0 
47. Connell, J. (2014). Measuring Quality of Life in Mental Health: Are we asking the right questions? Social Science and Medicine, 120:12-20.

https://doi.org/10.1016/j.socscimed.2014.08.026

48. Siggins Miller Consultants. (2003). Consumer self-rated outcome measures in mental health: A report to the Mental Health Branch, Department of Human Services. Retrieved from: http://www.health.vic.gov.au/mentalhealth/outcomes/csrfinal.pdf

49. Slade, M. (2002). What outcomes to measure in routine mental health services, and how to assess them: a systematic review. Australian and New Zealand Journal of Psychiatry, 36(6), 743-53. https://doi.org/10.1046/j.1440-1614.2002.01099.x

50. Reeve, B.B., Wyrwich, K.W., Wu, A.W., Velikova, G., Terwee, C.B., Snyder, “...”, Butt, Z. (2012). ISOQOL recommends minimum standards for patient-reported outcome measures used in patient-centered outcomes and comparative effectiveness research. Quality of Life Research, 22(8), 1889-1905. https://doi.org/10.1007/s11136-012-0344-y

51. World Health Organisation. (1993). WHOQOL Study Protocol: The Development of the World Health Organisation Quality of Life Assessment Instrument: Publication MNH/ PSF/93.9 Geneva, Switzerland: Division of Mental Health, WHO. https://doi.org/10.1007/BF00435734

52. Krägeloh C.U., Billington D.R., Hsu P.H-C, Feng X.J., Medvedev O.N., Kersten, P., “...”, \& Siegert, R.J. (2016). Ordinal-To-Interval Scale Conversion Tables and National Items for the New Zealand Version of the WHOQOL-BREF. PLoS ONE, 11(11), 1-15. https://doi.org/10.1371/journal.pone.0166065

53. Krägeloh, C.U., Kersten, P., Billington, D.R., Hsu, P.H., Shepherd, D., Landon, J., \& Feng, X J. (2012). Validation of the WHOQOL-BREF quality of life questionnaire for general use in New Zealand: confirmatory factor analysis and Rasch analysis. Quality of Life Research, 22(6), 1451-1457. https://doi.org/10.1007/s11136-012-0265-9 
54. Rethfeldt, S. (2007). Comparison of paper-and-pencil with online survey methodology using the quality of life instrument (WHOQOL-BREF) developed by the World Health Organisation (Unpublished BHSc(Hons) thesis), Auckland University of Psychology, Auckland, New Zealand.

55. Medvedev, O.N., Siegert, R.J., Kersten, P., Krägeloh, C.U. (2017). Improving the precision of the five factor Mindfulness questionnaire using a Rasch approach. Mindfulness, 8(4), 995-1008. http://dx.doi.org/10.1007/s12671-016-0676-8

56. Medvedev, O.N., \& Landhuis, C.E. (2018). Exploring constructs of well-being, happiness, and quality of life. PeerJ, 6:e4903. https://doi.org/10.7717/peerj.4903

57. Jacob, S., Munro, I., \& Taylor, B.J. (2017). Mental Health Recovery: A review of the peerreviewed published literature. The Australian Journal of Nursing Practice, Scholarship \& Research, 24(1), 53-61. https://doi.org/10.1016/j.colegn.2015.08.001

58. Ellison, M.L., Belanger, L.K., Niles, B.L., Evans, L.C. \& Bauer, M.S. (2018). Explication and definition of Mental Health Recovery: A systematic review. Administration and Policy in Mental Health and Mental Health Services Research, 45(1) 91-102. https://doi.org/10.1007/s10488-016-0767-9

59. Williams, K., Sansoni, J., Morris, D., Grootemaat, P., \& Thompson, C. (2016). Patientreported outcome measures: Literature Review. Australian Commission on Safety and Quality in Health Care. IBSN: 978-1-925224-74-0. Retrieved from: https://www.safetyandquality.gov.au/wp-content/uploads/2017/01/PROMs-Literature$\underline{\text { Review-December-2016.pdf }}$

60. Gordon, S.E., Ellis, P.M., Siegert, R.J. et al. (2013). Development of a Self-Assessed Consumer Recovery Outcome Measure: My Voice, My Life. Adm Policy Ment Health (2013) 40: 199. https://doi-org.ezproxy.aut.ac.nz/10.1007/s10488-012-0417-9 